\title{
Physicians' responses to computerized drug interaction alerts with password overrides
}

Yasuyuki Nasuhara ${ }^{1 *}$, Ken Sakushima ${ }^{2}$, Akira Endoh ${ }^{3}$, Reona Umeki ${ }^{3}$, Hiromitsu Oki ${ }^{4}$, Takehiro Yamada ${ }^{4}$, Ken Iseki ${ }^{4,5}$ and Makoto Ishikawa ${ }^{1}$

\begin{abstract}
Background: Although evidence has suggested that computerized drug-drug interaction alert systems may reduce the occurrence of drug-drug interactions, the numerous reminders and alerts generated by such systems could represent an excessive burden for clinicians, resulting in a high override rate of not only unimportant, but also important alerts.

Methods: We analyzed physicians' responses to alerts of relative contraindications and contraindications for coadministration in a computerized drug-drug interaction alert system at Hokkaido University Hospital. In this system, the physician must enter a password to override an alert and continue an order. All of the drug-drug interaction alerts generated between December 2011 and November 2012 at Hokkaido University Hospital were included in this study.

Results: The system generated a total of 170 alerts of relative contraindications and contraindication for coadministration; 59 (34.7\%) of the corresponding orders were cancelled after the alert was accepted, and 111 (65.3\%) were overridden. The most frequent contraindication alert was for the combination of 3-hydroxy-3-methylglutaryl-coenzyme A reductase inhibitors and fibrates. No incidents involving drug-drug interactions were reported among patients who were prescribed contraindicated drug pairs after an override.

Conclusions: Although computerized drug-drug interaction alert systems that require password overrides appear useful for promoting medication safety, having to enter passwords to override alerts may represent an excessive burden for the prescribing physician. Therefore, both patient safety and physicians' workloads should be taken into consideration in future designs of computerized drug-drug interaction alert systems.
\end{abstract}

Keywords: Adverse drug event, Alert fatigue, Computerized drug-drug interaction alert system, Contraindications for coadministration

\section{Background}

Numerous widespread patient safety issues were identified in a report published by the Institute of Medicine in 2000 entitled "To Err is Human" [1]. Many other reports have shown that among all medical incidents that occur in hospitals, drug-related incidents are the most prevalent [2-4]. Gandhi et al. reported that $25 \%$ of the outpatients at four adult primary care practices experienced adverse drug events (ADEs) in ambulatory care; $13 \%$ of these events were serious, $28 \%$ were ameliorable, and

\footnotetext{
* Correspondence: nasuhara@med.hokudai.ac.jp

1 Division of Hospital Safety Management, Hokkaido University Hospital, Sapporo, Japan

Full list of author information is available at the end of the article
}

$11 \%$ were preventable [5]. Unlike ADEs, which are often unpredictable, drug-drug interactions (DDIs) can be avoided if physicians take extra precautions when prescribing medications. Although evidence has shown that a computerized DDI alert system (DIAS) could reduce the incidence of DDIs [6-10], the burden of numerous reminders and alerts may cause clinicians to override not only unimportant, but also important alerts $[8,11]$. Some recent studies concluded that over-alerting and a lack of practical management or recommendations often cause physicians to disregard even serious alerts [12-14]. One review article concluded that between $49 \%$ and $96 \%$ of computerized DDI alerts are routinely ignored or overridden [15]. This "alert fatigue" strongly

\section{Biomed Central}

(C) 2015 Nasuhara et al. Open Access This article is distributed under the terms of the Creative Commons Attribution 4.0 International License (http://creativecommons.org/licenses/by/4.0/), which permits unrestricted use, distribution, and reproduction in any medium, provided you give appropriate credit to the original author(s) and the source, provide a link to the Creative Commons license, and indicate if changes were made. The Creative Commons Public Domain Dedication waiver (http://creativecommons.org/publicdomain/zero/1.0/) applies to the data made available in this article, unless otherwise stated. 
limits the applicability of automated alerts. A systematic review showed that conditions such as low specificity, low sensitivity, and unclear information content may make the alert system prone to errors that result in active failures of the physician, such as ignoring important alerts, misinterpretation, and incorrect handling [15]. With the goal of reducing alert fatigue, a number of studies have attempted to identify and rate key DDIs $[16,17]$. Findings from these studies suggest that physicians typically override alerts for the following three reasons: prior awareness of the DDI; insufficient knowledge of the DDI; or carelessness.

The DIAS at Hokkaido University Hospital has a very unique function in that when an alert of contraindications for coadministration is triggered, the physician must contact the hospital pharmacist to obtain a password; these passwords are randomly generated and changed daily. Next, to complete the order, the physician must enter the password along with a reason for the override. Therefore, physicians can override alerts only after considering the associated DDIs. To our knowledge, this is the first study to investigate override rates among physicians with awareness of DDIs using a DIAS that requires a password override.

To clarify physicians' responses, we examined all DDI alerts generated for one year at Hokkaido University Hospital. In addition, to utilize more homogenized subjects, we examined only alerts generated due to relative contraindications and contraindications for coadministration as described in drug package inserts. We also examined the profiles of DDIs with respect to drug types and the presence or absence of incidents involving DDIs.

\section{Methods \\ Drug-drug interaction database at Hokkaido University Hospital}

The DDI database at Hokkaido University Hospital was developed by the institution's pharmacy department. In Japan, drug package inserts must include the following information in relation to DDIs: 1) possible DDIs with other drugs have been considered; 2) the drug should not be coadministered with other drugs because serious DDIs may occur (relative contraindications for coadministration); and 3) coadministration of the drug with other drugs is prohibited due to the high prevalence of serious DDIs (contraindications for coadministration). The latter two points were manually entered into the computerized DIAS by the pharmacy department. Drug pairs in which contraindications were listed in the package insert of one drug but not the other were also included. The database was updated as new drugs were approved and drug package inserts were revised.

\section{Order entry system at Hokkaido University Hospital}

The DIAS at our hospital was originally developed by the Division of Medical Information Planning at Hokkaido University Hospital in collaboration with NEC (Nippon Denki). A computerized DIAS programmed with .NET and Visual Basic was incorporated into the existing order entry system. When a DDI is detected through cross-checking prescriptions with the DDI database, a pop-up alert window, as shown in Fig. 1 (in Japanese), is displayed in real time. An alert is triggered upon occurrence of any of the following situations: interactions with prescriptions ordered during the same visit; interactions with prescriptions ordered by different physicians; or interactions with previous prescriptions that are still active. In the case of relative contraindications and contraindications for coadministration, a "Contraindication!" message, accompanied by the reason for the alert, is displayed on the screen. If the physician wishes to override the alert and continue the order, they must first enter a password. To obtain the password, which is randomly generated and changed daily, they have to contact the hospital pharmacists. To proceed with the order, physicians must also enter the reason for overriding the alert by clicking corresponding buttons displayed on the monitor; these reasons include a long interval to avoid DDI, an expected DDI, an emergency situation in which the order is necessary, and planned use of a modified dosage to avoid the DDI. A flow chart of the computerized DIAS used at our hospital is shown in Fig. 2.

\section{Data collection and analysis}

Hokkaido University Hospital is a national university hospital in Japan with 936 beds, about 3,000 outpatients/

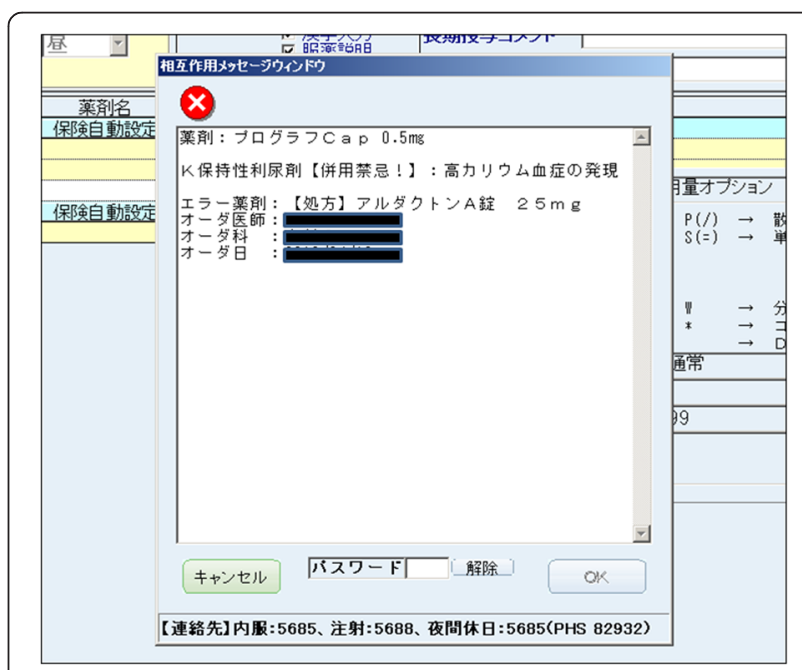

Fig. 1 The pop-up window displayed on the screen in real time when an alert of relative contraindications and contraindications for coadministration is generated. A "Contraindication!" message is displayed on the screen along with the reason for the alert. The protocol for requesting a password is also shown 


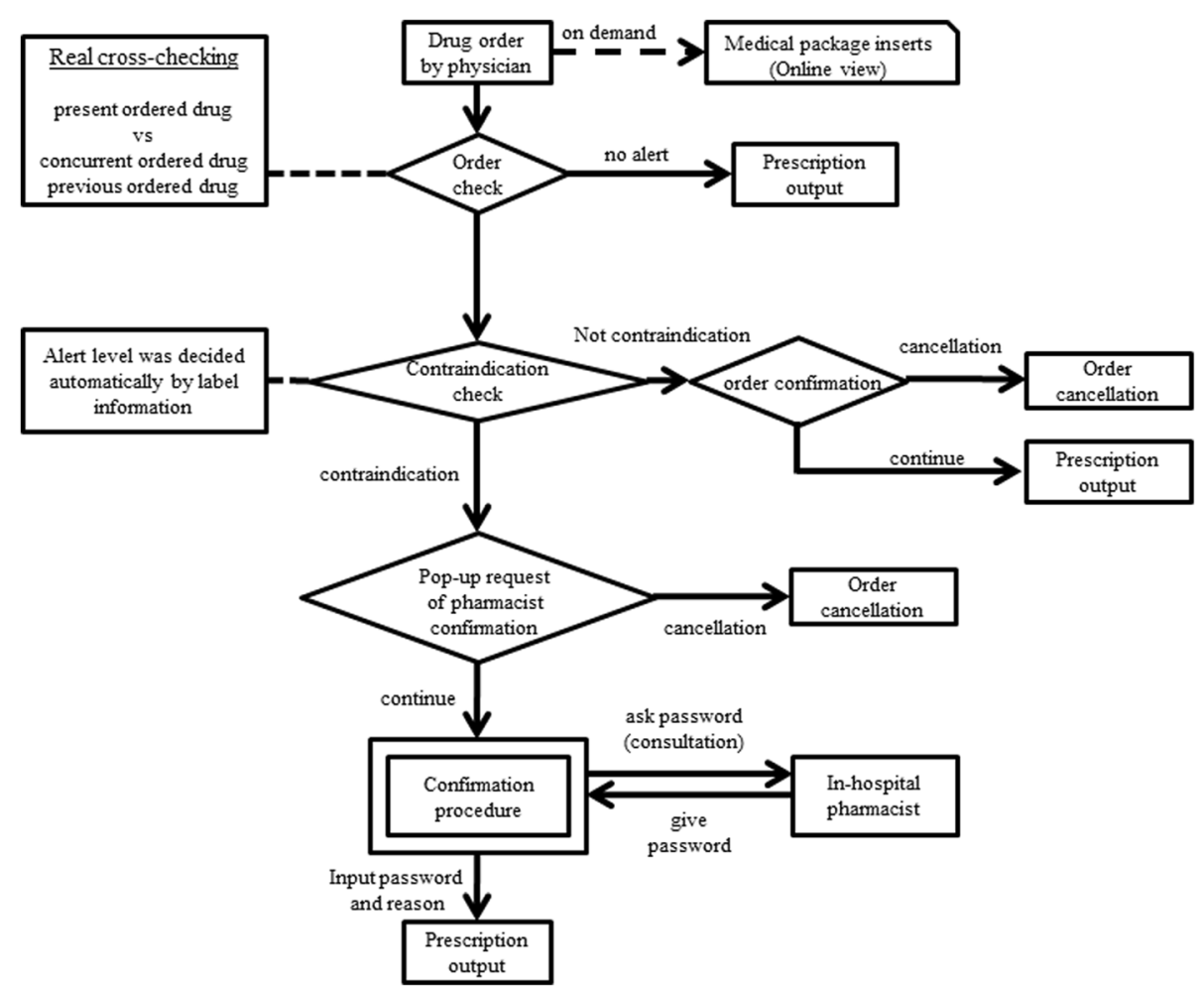

Fig. 2 A flow chart showing the computerized drug-drug interaction alert system used at Hokkaido University Hospital

day, about 550 doctors, and an average of 37,500 prescriptions/month. All drugs prescribed at our hospital are ordered through the computerized order entry system and recorded in the database; all DDI alerts triggered by the system are also recorded. All DDI alerts triggered by the computerized DIAS for prescriptions from Hokkaido University Hospital between December 2011 and November 2012 were included in this study (268,622 prescriptions for the study period). A database specialist (RU) from the Division of Medical Information Planning extracted and compiled the necessary data into a spreadsheet for analysis by two experienced ( $\mathrm{KN}$ and YN). In April 2012 and August 2014, medical records were reviewed $(\mathrm{YN})$ for the presence or absence of incidents involving DDIs in patients prescribed contraindicated drug pairs.

\section{Ethics}

This study was approved by the Institutional Review Board of Hokkaido University Hospital.

\section{Results}

For one year from December 2011 to November 2012, 1449 alerts for contraindicated coadministration were recorded in the system log (Fig. 3). Among these alerts, some were generated for the same patient and the same drug pairs ordered by the same physician on the same day. Physicians had two typical response patterns when making repeated attempts to reorder the prescription. The first response primarily involved physicians who were unfamiliar with the DIAS. These physicians thought that they could override DDI alerts simply by clicking the "cancel" button. Once discovering that their order had

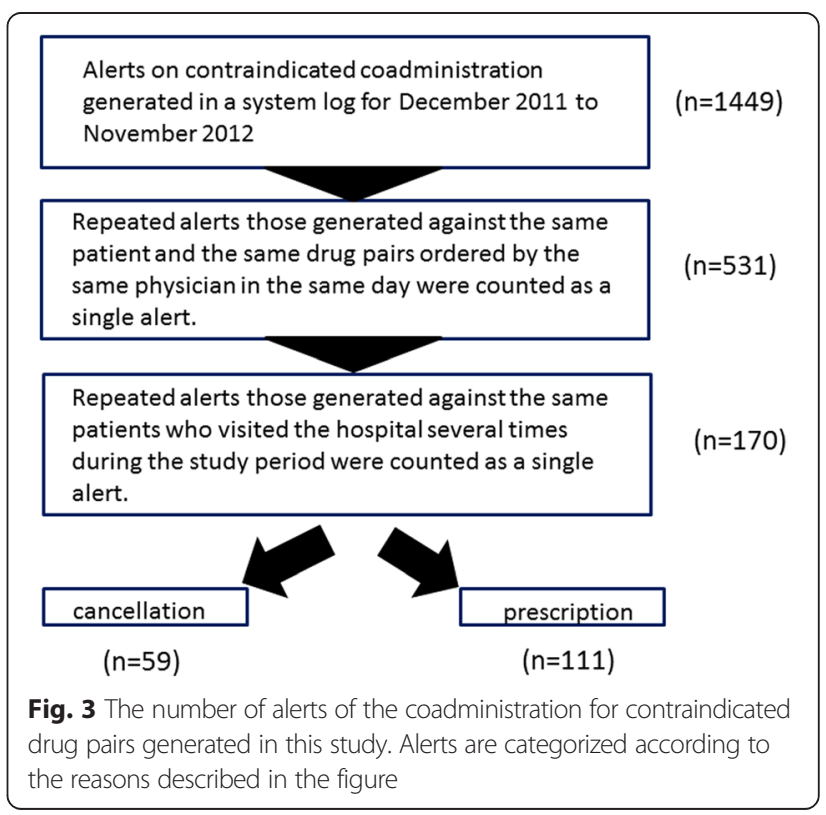


actually been cancelled, they would reorder the drugs and contact the hospital pharmacist to obtain a password. The other common response by physicians was to purposefully cancel their order when a DDI alert was triggered. Then, after careful consideration, they would then reorder the same pair of contraindicated drugs and contact the pharmacist to obtain a password. When these types of repeated alerts were counted as single alerts, the number of alerts for the study period dropped from 1449 to 531 . In addition, some patients visited the hospital several times during the study period. Therefore, alerts generated for the same drug pair for the same patient by the same physician were also counted as single alerts; this further reduced the number of alerts generated during the study period to 170 .

In response to these 170 alerts, 59 (34.7\%) of the corresponding orders were cancelled due to alert acceptance, and 111 (65.3\%) were prescribed after a password override (Fig. 3). Drug pairs for which physicians overrode DDI alerts more than five times are listed in Table 1. The most frequent contraindication alert was for the combination of HMG-CoA (3-hydroxy-3-methylglutaryl-coenzyme A) reductase inhibitors and fibrates, followed by tacrolimus hydrate and potassium-sparing diuretics. No incidents due to these combinations were recorded in the patients' medical records up to 28 months after the prescriptions were issued. Drug combinations that were cancelled more than twice due to alert acceptance are listed in Table 2.

\section{Discussion}

In the present study, the overall alert override rate was $65.3 \%$, which is compatible with override rates reported in previous studies between $49 \%$ and $96 \%$ [15]. One study reported an overall acceptance rate of only $8.5 \%$; however, most of the alerts were overridden (91.5\%). Regarding the recognition of DDIs in that study, physicians were aware of $82.0 \%$ of the DDIs, were unaware of $15.9 \%$, and ignored $2.1 \%$ of the alerts [18]. The present study has three important differences from previous studies. First, in order to override alerts, physicians

Table 1 Drug pairs for which physicians overrode drug-drug interaction alerts more than five times

\begin{tabular}{|c|c|c|}
\hline Drug pairs & & Orders \\
\hline HMG-CoA reductase inhibitors & Fibrates & 35 \\
\hline Tacrolimus hydrate & Potassium-sparing diuretics & 15 \\
\hline Triptans & Triptans $^{a}$ & 8 \\
\hline Immunosuppressants & Immunosuppressants & 7 \\
\hline Albumin tannate & Ferrous fumarate & 7 \\
\hline \multirow[t]{2}{*}{ Potassium-sparing diuretics } & Potassium-sparing diuretics & \\
\hline & Potassium chloride & 6 \\
\hline
\end{tabular}

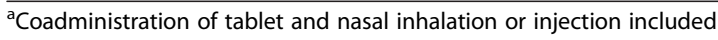

Table 2 Drug combinations cancelled due to alert acceptance

\begin{tabular}{|c|c|c|}
\hline Drug pairs & & Orders \\
\hline Albumin tannate & Ferrous fumarate & 10 \\
\hline HMG-CoA reductase inhibitors & Fibrates & 8 \\
\hline Miconazole & Calcium channel blockers & 5 \\
\hline Voriconazole & Triazolam & 4 \\
\hline Triptans & Triptans $^{a}$ & 4 \\
\hline Selegiline hydrochloride & $\mathrm{SSRI}^{\mathrm{b}}$ & 3 \\
\hline \multirow[t]{2}{*}{ Potassium-sparing diuretics } & Potassium-sparing diuretics & \\
\hline & Potassium chloride & 3 \\
\hline
\end{tabular}

Combinations that were cancelled more than twice are listed

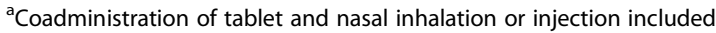

${ }^{\text {b}}$ SSRI: selective serotonin reuptake inhibitor

had to contact pharmacists to obtain passwords, which were randomly assigned and changed daily. When physicians see alerts on the screen, they may intentionally or unintentionally ignore them and click the "continue" or "ignore" button. In the computerized DIAS in our hospital, the password system prevents physicians from prescribing contraindicated drug pairs carelessly. Therefore, physicians who overrode alerts and prescribed all 111 contraindicated drug pairs were aware of the contraindications. Second, we only analyzed alerts generated due to relative contraindications and contraindications for coadministration as described in drug package inserts. Third, because physicians had to consult with pharmacists directly to obtain override passwords, physicians could only decide to continue with their orders after receiving detailed pharmaceutical reasons for the contraindications. The present study therefore revealed that even under such conditions, physicians still override DDI alerts at a high rate. Furthermore, no ADEs related to DDIs were reported among patients who were prescribed contraindicated drug pairs after an alert override.

On the other hand, $34.7 \%$ of all contraindicated orders were cancelled due to DDI alerts. In these cases, the physician may have cancelled the order because they were unaware of the contraindication before the alert. They then could have decided that the contraindicated drugs were not absolutely necessary and prescribed alternative medications. The cancelled drug pairs were not only low-risk pairs such as albumin tannate and ferrous fumarate, but also high-risk pairs such as voriconazole and triazolam (Table 2). As a result, it is also possible that alerts help prevent incidents involving DDIs. Therefore, we conclude that a computerized DIAS that requires password overrides for coadministration of contraindicated drugs is useful for promoting medication safety.

This study did have several limitations. First, we only performed quantitative analysis. In the DIAS at our hospital, physicians choose the reason for overriding an alert 
by clicking corresponding buttons displayed on the monitor; these reasons include a long interval to avoid DDI, an expected DDI, an emergency situation in which the order is necessary, planned use of a modified dosage to avoid the DDI, and others. To understand the behavior of physicians, it is important to analyze the frequency of selection for each reason; however, in this study, nearly all of the reasons were "others", which may indicate negligence on the part of the physician. Therefore, we could not perform an analysis regarding the reasons for alert overrides; such an analysis should be performed in a future study. Second, detailed information about physicians, such as their clinical experience or whether they were generalists or specialists, was not obtained in this study. The influence of background characteristics and experience on physicians' behaviors in response to alerts should be investigated in a future study. It would also be important to analyze physicians' behaviors after obtaining detailed explanations from pharmacists. The DIAS recorded all instances in which passwords were issued, but not consultations between physicians and pharmacists; therefore, when physicians attempted to override alerts, we could not distinguish whether they had obtained detailed pharmaceutical explanations before cancelling the order. It would also be useful to obtain physicians' voices regarding systems that require password overrides to continue an order and how such systems affect their workflow.

In a recent report, contraindicated combinations based on drug package inserts were prescribed for just $0.2 \%$ of over 400,000 patients in a Japanese database of health insurance claims [19]. The most frequently contraindicated drug combinations in that study involved lipid-regulating drugs, anti-migraine drugs, drugs that included metal irons, antifungals, macrolides, and immunosuppressants. Therefore, the result of our study may be generalized in point of the sort of prescribed contraindicated coadministration pairs at least in Japan. Furthermore, unlike the study involving patients in the Japanese database of health insurance claims, our study investigated the presence or absence of incidents involving DDIs.

These results suggest that physicians' workloads should be considered in future computerized DIAS designs. One method of reducing alert fatigue may be to temporarily deactivate alerts for the same contraindicated drug pairs prescribed to the same patient by the same physician that have been overridden. The results of this study shed light on possible strategies for developing a more efficient DIAS.

\section{Conclusions}

In this study, we explored the behavior of physicians only in regard to orders requiring password overrides for the coadministration of contraindicated drug pairs in a DIAS at Hokkaido University Hospital in Japan. Based on our results and those from previous studies, a computerized DIAS with password overrides is useful for promoting medication safety. However, the password override system may represent an excessive burden for physicians and thereby increase alert fatigue. Even though physicians who were aware of contraindicated drug pairs still overrode DDI alerts at a high rate, no incidents involving DDIs were reported among patients who were prescribed contraindicated drug pairs after an override. On the other hand, $34.7 \%$ of the contraindicated orders were cancelled due to alert acceptance. Therefore, both patient safety and physicians' workloads should be considered in future computerized DIAS designs.

\section{Abbreviations \\ ADE: Adverse Drug Event; DDI: Drug-Drug interaction; DIAS: Drug-Drug Interaction Alert System.}

\section{Competing interests}

The authors declare that they have no competing interests.

\section{Author's contributions}

YN and KS conceived the study idea and design. $K S, A E$, and RU were responsible for data collection. YN, KS, HO, and TY were responsible for the interpretation of data. YN and KS participated in writing and $\mathrm{Kl}$ and $\mathrm{MI}$ critically reviewed the manuscript. All authors read and approved the final manuscript.

\section{Acknowledgements}

The authors would like to thank Prof. Yoichi Ito for his advice early in the study.

\section{Author details}

'Division of Hospital Safety Management, Hokkaido University Hospital, Sapporo, Japan. ${ }^{2}$ Department of Regulatory Science, Hokkaido University Graduate School of Medicine, Sapporo, Japan. ${ }^{3}$ Division of Medical Information Planning, Hokkaido University Hospital, Sapporo, Japan. ${ }^{4}$ Department of Pharmacy, Hokkaido University Hospital, Sapporo, Japan. ${ }^{5}$ Laboratory of Clinical Pharmaceutics and Therapeutics, Faculty of Pharmaceutical Sciences, Hokkaido University, Sapporo, Japan.

Received: 1 April 2015 Accepted: 23 July 2015

Published online: 28 August 2015

\section{References}

1. Institute of Medicine (US) Committee on Quality of Health Care in America. To Err is Human: Building a Safer Health System. In: Kohn LT, Corrigan J, Donaldson MS, editors. Washington DC: National Academy Press; 2000.

2. Lazarou J, Pomeranz BH, Corey PN. Incidence of adverse drug reactions in hospitalized patients: a meta-analysis of prospective studies. JAMA. 1998;279:1200-5.

3. An Organization with a Memory: Report of an Expert Group on Learning from Adverse Events in the NHS. Chaired by the Chief Medical Officer. London: The Stationary Office Limited, 2000

4. Bates DW, Cullen DJ, Laird N, Peterson LA, Small SD, Servi D, et al. Incidence of adverse drug events and potential adverse drug events: Implications for prevention. JAMA. 1995;274:29-34.

5. Gandhi TK, Weingart SN, Borus J, Seger AC, Peterson J, Burdick E, et al. Adverse drug events in ambulatory care. N Engl J Med. 2003;348:1556-64.

6. Kaushal R, Shojania KG, Bates DW. Effects of computerized physician order entry and clinical decision support systems on medication safety. Arch Intern Med. 2003;163:1409-16.

7. Raschke RA, Gollihare B, Wunderlich TA, Guidry JR, Leibowitz Al, Peirce JC, et al. A computer alert system to prevent injury from adverse drug events: development and evaluation in a community teaching hospital. JAMA. 1998;280:1317-20. 
8. Glassman PA, Simon B, Belperio P, Lanto A. Improving recognition of drug interactions: benefits and barriers to using automated drug alerts. Med Care. 2002;40:1161-71.

9. Hunt DL, Haynes RB, Hanna SE, Smith K. Effects of computer-based clinical decision support systems on physician performance and patient outcomes: a systematic review. JAMA. 1998;280:1339-46.

10. Rind DM, Safran C, Phillips RS, Wang Q, Calkins DR, Delbanco TL, et al. Effect of computer-based alerts on the treatment and outcomes of hospitalized patients. Arch Intern Med. 1994;154:1511-17.

11. Hsieh TC, Kuperman GJ, Jaggi T, Hojnowski-Diaz P, Fiskio J, Williams DH, et al. Characteristics and consequences of drug allergy alert overrides in a computerized physician order entry system. J Am Med Inform Assoc. 2004;11:482-91.

12. Van der Sijs H, Aarts J, van Gelder T, Berg M, Vulto A. Turning off frequently overridden drug alerts: limited opportunities for doing it safely. J Am Med Inform Assoc. 2008;15:439-48.

13. Van der Sijs H, Mulder A, van Gelder T, Aarts J, Berg M, Vulto A. Drug safety alerts generation and overriding in a large Dutch university medical centre. Pharmacoepidem Dr S. 2009;118:941-7.

14. Mille F, Schwartz C, Brion F, Fontan JE, Bourdon O, Degoulet P, et al. Analysis of overridden alerts in a drug-drug interaction detection system. Int J Qual Health Care. 2008;20:400-5.

15. van der Sijs H, Aarts J, Vulto A, Berg M. Overriding of drug safety alerts in computerized physician order entry. J Am Med Inform Assoc. 2006;13:138-47.

16. Phansalkar S, Desai AA, Bell D, Yoshida E, Doole J, Czochanski M, et al. High priority drug-drug interactions for use in electronic health records. J Am Med Inform Assoc. 2012;19:735-43.

17. Phansalkar S, van der Sijs TAD, Desai AA, Bell DS, Teich JM, Middleton B, et al. Drug-drug interactions that should be non-interruptive in order to reduce alert fatigue in electronic health records. J Am Med Inform Assoc. 2013:20:489-93.

18. Yeh ML, Chang YJ, Wang PY, Li YC, Hsu CY. Physicians' responses to computerized drug-drug interaction alerts for outpatients. Comput Meth and Prog Bio. 2013;111:17-25.

19. Seki M, Tsubaki $H$. Drug utilization study of potential drug-drug interactions using the sampling dataset of the national database of health insurance claim information. An Official Journal of the Japan Association for Medical Informatics. 2014;34:293-304. abstract in English.

\section{Submit your next manuscript to BioMed Central and take full advantage of:}

- Convenient online submission

- Thorough peer review

- No space constraints or color figure charges

- Immediate publication on acceptance

- Inclusion in PubMed, CAS, Scopus and Google Scholar

- Research which is freely available for redistribution 